\title{
Application of a recombinant replicase to localize the Trionyx sinensis hemorrhagic syndrome virus and evaluate its effects on antiviral genes of $T$. sinensis
}

\author{
Sunjian LYU ${ }^{1}$, Xuemei YUAN ${ }^{1}$, Li LIU ${ }^{2 \bowtie}$, Haiqi ZHANG ${ }^{1 \bowtie}$, Zhe YU ${ }^{1}$, Xiaoying HANG ${ }^{1}$, Weida SHI ${ }^{1}$, \\ Yinglei $\mathrm{WU}^{1}$ \\ ${ }^{1}$ Agriculture Ministry Key Laboratory of Healthy Freshwater Aquaculture, Key Laboratory of Fish, Health and Nutrition of Zhejiang Province, \\ Zhejiang Institute of Freshwater Fisheries, Huzhou 313001, China \\ ${ }^{2}$ Institute of Hydrobiology, Zhejiang Academy of Agricultural Sciences, Hangzhou 310021, China
}

\begin{abstract}
Trionyx sinensis Hemorrhagic Syndrome Virus (TSHSV) is an arterivirus newly discovered in Chinese softshell turtles. Little is known about the effect of antibodies against the virus or the distribution of the virus in different organs of infected turtles. In this study, a partial protein of TSHSV-HP4 was produced using a prokaryotic expression system, and its polyclonal antibody was generated. The polyclonal antibody was confirmed by western blot and dot enzyme-linked immunosorbent assay (dot-ELISA). The distribution of TSHSV in different organs of $T$. sinensis was examined by immunohistochemistry (IHC) and the expression of immune-related genes was analyzed using quantitative real-time polymerase chain reaction (qRT-PCR). The results indicated that the recombinant TSHSV-HP4 protein was successfully expressed, and the generated polyclonal antibody showed specific binding to viral particles in the lung tissues of infected turtles. The IHC assay indicated that the virus was highly localized in various cells, including intestinal lymphocytes, enterocytes, kidney epithelial cells, spleen cells, lung macrophages, and cardiomyocytes. The qRT-PCR analysis revealed that TSHSV was detected in all organs tested, including the lungs, liver, kidneys, spleen, and heart. The numbers of viral mRNA copies in lung and heart tissues were significantly higher in the virus-antibody group than in the virus group. The interferonstimulated genes (ISGs), myxovirus resistance protein $2(M X 2)$ and radical $S$-adenosyl methionine domain containing 2 (RSAD2) were highly upregulated in all groups of infected turtles. Antibody-dependent enhancement (ADE) seemed to occur after stimulation by the polyclonal antibody, because significantly greater expression of the two genes was detected in the virus-antibody group than in the virus group. Overall, these results are important in understanding the cell localization of TSHSV and the immune response of infected turtles.
\end{abstract}

Key words: Trionyx sinensis Hemorrhagic Syndrome Virus (TSHSV); Replicase; Virus localization; Immune genes

\section{Introduction}

As one of the most important aquatic species in China, the Chinese softshell turtle (Trionyx sinensis) is widely cultured for food production, with output reaching up to 319081 tons in 2018 (Fishery Administration Bureau of Ministry of Agriculture and Rural Areas et al., 2018). However, recently $T$. sinensis has

\footnotetext{
\i LIU, Liuli6655@hotmail.com

Haiqi ZHANG, zmk407@126.com

(D) Sunjian LYU, https://orcid.org/0000-0001-7862-3097
}

Received Aug. 28, 2020; Revision accepted Dec. 6, 2020; Crosschecked Mar. 11, 2021

(C) Zhejiang University Press 2021 suffered from a serious viral disease caused by a new arterivirus named T. sinensis Hemorrhagic Syndrome Virus (TSHSV) (Liu et al., 2015). In 2013, this virus was first discovered in China, and now it is widespread in both young and adult commercially breeding $T$. sinensis. The related disease is highly contagious and lethal (Liu et al., 2015). This virus causes a fatal interstitial pneumonia and hyperemic laryngeal mucosa, which both lead to difficulties in breathing and death. Microscopic and gross lesions both indicate serious hemorrhaging in multiple organs of infected turtles (Liu et al., 2017). TSHSV belongs to the genus Arteriviridae and the unclassified family Arteriviridae, which also includes Porcine Reproductive and Respiratory Syndrome Virus 
(PRRSV), Equine Arteritis Virus (EAV), and Simian Hemorrhagic Fever Virus (SHFV) (Lyu et al., 2020). Similar to these arteriviruses, TSHSV comprises a large single-stranded RNA genome of positive polarity. The complete genome has been determined to be $17.9 \mathrm{~kb}$ in length. Eight hypothetical proteins (HPs) are speculated to be encoded by the viral genome (Lyu et al., 2019). These HPs were calculated as $79,308,1552$, 1692, 169, 194, 329, and 714 aa, respectively (Lyu et al., 2019). Among them, TSHSV-HP2 is similar to papainlike protease 2, while TSHSV-HP3 is a non-structural protein with serine-type endopeptidase activity and might be a replicase protein (Lyu et al., 2019). As a nonstructural protein (NSP), TSHSV-HP4 is predicted to have replicase polyprotein activity, and belongs to the P-loop containing nucleoside triphosphate hydrolase or endoribonuclease homologous superfamily (Lyu et al., 2019). The functions of the unclassified hypothetical viral proteins, TSHSV-HP1, TSHSV-HP5, TSHSVHP6, and TSHSV-HP7, are still unclear (Lyu et al., 2019). In arteriviruses, the replicase polyproteins are the key enzymes for RNA synthesis. These replicative enzymes are encoded in open reading frame 1a (ORF1a) and ORF1b, particularly the viral RNA-dependent RNA polymerase and RNA helicase (Fang and Snijder, 2010). The transmembrane NSPs are incorporated into the cellular organelles, particularly the endoplasmic reticulum, where early viral RNA synthesis occurs, resulting in increased expression of replicase proteins (Knoops et al., 2008).

Considering the importance of replicase polyprotein in arterivirus replication, the aim of this study was to clone and express a partial protein of the replicase polyprotein, namely TSHSV-HP4, in the Escherichia coli prokaryotic expression system. A polyclonal antibody against the viral replicase polyprotein was subsequently prepared to determine the localization of the virus in different organs and its effects on activating the immune system of $T$. sinensis.

\section{Materials and methods}

\subsection{Animal cultivation}

Chinese softshell turtles (T. sinensis) of about $500 \mathrm{~g}$ body weight were gathered from a livestock farm in Jiaxin, Zhejiang Province, China. Turtles were temporarily raised at $(25 \pm 2)^{\circ} \mathrm{C}$ in $400 \mathrm{~L}$ tanks, and were fed with commercial fodder. They were acclimated for one week prior to the experiments. The animal experiments were approved by Zhejiang Institute of Freshwater Fisheries Committee for Experimental Animal Management (Huzhou, China).

\subsection{Protein expression and polyclonal antibody preparation}

\subsubsection{PCR amplification of target sequence}

TSHSV-infected T. sinensis was used for viral RNA extraction. The lungs (previously determined to contain the highest copy number of TSHSV RNAs) were sampled from sacrificed sick turtles and the total RNA was extracted using RNAiso Plus (TaKaRa, Japan), following the manufacturer's procedure (Liu et al., 2015). The first-strand complementary DNA (cDNA) was synthesized from the total RNA using the M-MLV Reverse Transcriptase System (Promega, USA), according to the manufacturer's protocol. The target segment was amplified using specific primers TSHSV-66101F (5'CGCGGATCCGCGATGGCTAGCATCCTTT-3') and TSHSV-66101R(5'-CCGCTCGAGCGGTTATACTTGT TCAAATTCAGG-3') with BamHI and XhoII (enzyme digestion sites underlined), respectively. Primer was based on the sequence of TSHSV-HP4 (GenBank accession No. MH447987.1). Polymerass chain reaction (PCR) amplification was performed using OneTaq QuickLoad $2 \times$ Master Mix (NEB, USA) in $25 \mu \mathrm{L}$ of reaction buffer, making up as follows: $12 \mu \mathrm{L}$ Mix, $8.5 \mu \mathrm{L}$ PCR grade water, $2.5 \mu \mathrm{L}$ cDNA, and $1 \mu \mathrm{L}(10 \mu \mathrm{mol} / \mathrm{L})$ of each primer. The protocol for reaction was denaturing at $95{ }^{\circ} \mathrm{C}$ for $10 \mathrm{~min}$, followed by cycling 35 times at $95{ }^{\circ} \mathrm{C}$ for $30 \mathrm{~s}, 58{ }^{\circ} \mathrm{C}$ for $45 \mathrm{~s}$ and $72{ }^{\circ} \mathrm{C}$ for $1 \mathrm{~min}$, and finally an extension process at $72{ }^{\circ} \mathrm{C}$ for $5 \mathrm{~min}$. The PCR amplification products were analyzed by electrophoresis on $1.2 \%(0.012 \mathrm{~g} / \mathrm{mL})$ agarose gels, and the target segment (TSHSV-661) was extracted using a DNA gel extraction kit (TIANGEN Biotech, China).

2.2.2 Construction of TSHSV-HP4 gene expression vector

The purified TSHSV-661 segment was digested using Bam HI and XhoII restriction enzymes at $37{ }^{\circ} \mathrm{C}$ for $3 \mathrm{~h}$ and the digested products were inserted into the pET-30a vector (TaKaRa, China) via a ligase enzyme, and the recombinant plasmid was named as pET30a-TSHSV-661. The recombinant plasmid was 
transformed directly into Escherichia coli BL21 (DE3) cells (TaKaRa, Japan). The successful construction of the pET30a-TSHSV-661 clone was confirmed by PCR amplification using the above specific primers by the same PCR reaction, and amplified products were checked via agarose gel electrophoresis. The plasmid of the positive pET30a-TSHSV-661 clone was extracted for sequencing (Sunya, China).

2.2.3 Expression and purification of recombinant TSHSV-HP4 protein, and preparation of the corresponding polyclonal antibody

For TSHSV-HP4 protein production, the BL21 (DE3)/pET30a-TSHSV-661 cells were cultured in Luria-Bertani (LB) medium at $37{ }^{\circ} \mathrm{C}$ until the optical density at $600 \mathrm{~nm}\left(\mathrm{OD}_{600}\right)$ reached about 0.4 , after which $1 \mathrm{mmol} / \mathrm{L}$ isopropyl thiogalactoside (IPTG) was added, and the incubation process continued for $4 \mathrm{~h}$ at $26^{\circ} \mathrm{C}$. The protein was affinity-purified under denaturing conditions using Ni-NTA Spin Kit (Qiagen, USA). Sodium dodecyl sulfate-polyacrylamide gel electrophoresis (SDS-PAGE) was performed with a $15 \%$ (volume fraction) gel to determine TSHSV-HP4 protein expression. The purified TSHSV-HP4 protein was used for raising rabbit anti-TSHSV-HP4 polyclonal antibody (Huabio, China).

\subsection{Western blot assay}

The purified TSHSV-HP4 protein was quantified and separated by SDS-PAGE. Separated protein was then transferred onto a nitrocellulose membrane according to the standard method. After transfer, membranes were rapidly washed with phosphate-buffered saline (PBS) and blocked with QuickBlock ${ }^{\mathrm{TM}}$ blocking buffer (Beyotime, China) for $15 \mathrm{~min}$. Subsequent primary antibody incubation was conducted using rabbit anti-His antibody (1:2000, volume ratio, the same below) and rabbit anti-TSHSV-HP4 polyclonal antibody $(1: 1000)$ overnight at $4{ }^{\circ} \mathrm{C}$. This was followed by incubating for $1 \mathrm{~h}$ with goat anti-rabbit IgG conjugated with horseradish peroxidase (HRP; 1:2000) at room temperature. Blots were revealed with enhanced chemiluminescence using BeyoECL Star (Beyotime, China).

\subsection{Dot-ELISA and tissue blot-ELISA}

Dot enzyme-linked immunosorbent assay (dotELISA) and tissue blot-ELISA for TSHSV detection were performed as previously described with slight modification (Chen et al., 2017). The total protein in the lungs from wild and laboratory TSHSV-infected turtles was extracted using radio immune precipitation assay (RIPA) lysis buffer containing $1 \%$ phenylmethylsulfonyl fluoride (PMSF), according to the operation manual. The extracted proteins were stored at $-80{ }^{\circ} \mathrm{C}$ until used. The blood from TSHSV-infected turtles was placed in an enzyme-free plate and allowed to stand at $4{ }^{\circ} \mathrm{C}$ overnight. Next, the top serum was collected after triturating the clotted blood, and stored at $-80^{\circ} \mathrm{C}$. Purified TSHSV-HP4 protein and the protein extracted from the lungs of healthy turtles were used as the positive and negative controls, respectively. About $2 \mu \mathrm{L}$ of each sample was individually spotted onto nitrocellulose membranes (Amersham Biosciences, UK) and air-dried. The membranes were soaked in QuickBlock ${ }^{\mathrm{TM}}$ blocking buffer (Beyotime, China) for $15 \mathrm{~min}$, followed by incubation in diluted polyclonal antibody solutions (1:100) for $1 \mathrm{~h}$ at room temperature. The membranes were then washed three times with PBST $(0.01 \mathrm{~mol} / \mathrm{L} \mathrm{PBS,} 0.05 \%$ Tween-20, pH 7.4), and incubated in diluted goat antirabbit immunoglobulin $\mathrm{G}(\mathrm{IgG})(1: 10000)$ conjugated with HRP solution for $1 \mathrm{~h}$ at room temperature. The membranes were finally incubated in 3,3'-diaminobenzidine (DAB) solution for $30 \mathrm{~min}$ for color development.

\subsection{Immunohistochemistry analysis of viral localization}

After crude TSHSV- and PBS-challenge for $3 \mathrm{~d}$, the turtles with clinical symptoms were sacrificed and their small intestines, lungs, liver, spleen, kidneys, and heart were collected for Immunohistochemistry (IHC) analysis. Organs were fixed in $4 \%$ paraformaldehyde, embedded in paraffin wax, and sectioned using routine procedures (Lyu et al., 2020).

\subsection{Histopathological analysis of lung tissue}

The lungs were dissected from the turtles infected with TSHSV and then fixed in 4\% paraformaldehyde. Samples were embedded in paraffin wax and cut into 6 - $\mu \mathrm{m}$ slices for hematoxylin and eosin (H\&E) staining. Histopathological features, especially the inflammation responses, were observed and compared.

\section{7 qRT-PCR analysis of immunity genes}

The prepared polyclonal antibody against TSHSVHP4 was mixed with crude TSHSV at room temperature for $10 \mathrm{~min}$ prior to injections. Five turtles in each group 
received an intramuscular injection of $200 \mu \mathrm{L}$ crude virus (corresponding to $2 \times 10^{7}$ virus copies; virus group), $400 \mu \mathrm{L}$ mixture containing $200 \mu \mathrm{L}$ polyclonal antibody and $200 \mu \mathrm{L}$ crude virus (virus-antibody group), $200 \mu \mathrm{L}$ polyclonal antibody (antibody group), or $200 \mu \mathrm{L} \mathrm{PBS} \mathrm{(control} \mathrm{group).}$ After the 3-d challenge, the turtles were sacrificed and the lungs, liver, kidneys, spleen, and heart from each turtle were collected for total RNA extraction. The total RNA was extracted using RNAiso Plus (TaKaRa, Japan), according to the manufacturer's protocol. cDNA synthesis and qRT-PCR were performed according to the methods mentioned in our previous research (Lyu et al., 2020). Specific primers for the virus and immune-related genes, including myxovirus resistance protein 2 (MX2) and radical $S$-adenosyl methionine domain containing 2 (RSAD2), are shown in Table 1. The messenger RNA (mRNA) expression levels of viral and immune-related genes were quantified by the standard $2^{-\Delta \Delta C_{\mathrm{T}}}$ method after normalizing to $\beta$-actin.

\subsection{Statistical analysis}

The data were presented as mean \pm standard error (SE). All experimental data were analyzed using one-way analysis of variance (ANOVA) to determine the differences among different groups. Statistical significance was defined by $P<0.05$.
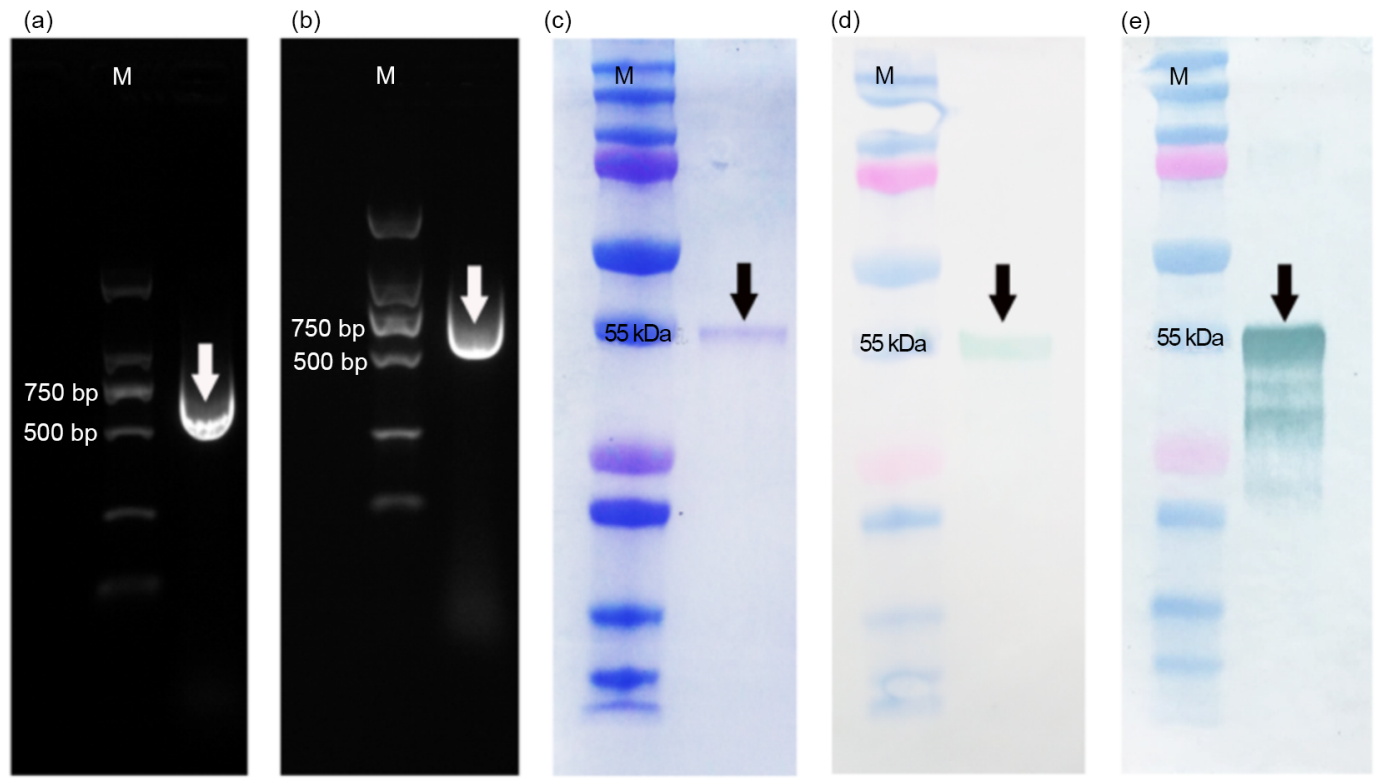

Fig. 1 PCR amplification and western blot assay of target TSHSV-HP4. The arrows indicate the target PCR product amplified from the synthesized cDNA using specific primers (a) and a positive clone containing pET30a-TSHSV-661 plasmid (b). (c) SDS-PAGE analysis of the purified TSHSV-HP4 protein. Western blot assay of the expressed partial TSHSV-HP4 protein using a monoclonal antibody against $6 \times$ His tag (d) and the prepared polyclonal antibody against the purified TSHSV-HP4 protein (e). M: marker. 
(Fig. 1c). Western blotting assay showed that the prepared polyclonal antibody could specifically bind to the purified protein using the anti-His tag antibody (Fig. 1d) and anti-rabbit antibody (Fig. 1e).

\subsection{Detection of TSHSV using dot-ELISA and tissue blot-ELISA}

Based on the dot-ELISA, a strong positive reaction was detected in dots prepared using purified recombinant TSHSV-HP4 protein (Fig. 2). To determine if tissue blot-ELISA could be used to detect TSHSV in tissue and serum samples from TSHSV-infected turtles, proteins extracted from the lungs of wild and laboratory TSHSV-infected turtles and serum derived from laboratory TSHSV-infected turtles were tested with the recombinant TSHSV-HP4 polyclonal antibody. Strong reactions were observed with the protein extracts from TSHSVpositive lungs, weak positive reactions with the serum extracts, and a negative reaction with extracts from the control lung tissues.

\subsection{Assay of TSHSV localization in different organs}

To confirm the viral cytopathic effects and localization, an IHC assay was conducted in selected tissues. A strong positive reaction against the viral protein was observed in five tissues: the small intestine, lung, liver, spleen, and kidney (Fig. 3). No IHC-positive staining for virus protein was observed in the hearts of infected turtles or in those of the negative control group. In the small intestines, a positive signal was observed in the lymphocytes and epibiotic enterocytes in the intestinal villi of infected turtles, compared to those of the negative control group. The mucous epithelial cells of the intestine displayed necrocytosis and abscission with serious nuclear shrinkage and significant lymphocyte accumulation. In the kidneys, a clear signal of virus protein was evident in the glomerulus and epithelial cells, while sporadic signals were observed within the lumen of tubules and in the necrotic debris. Additionally, a clear signal was observed in the white pulp, but not in the

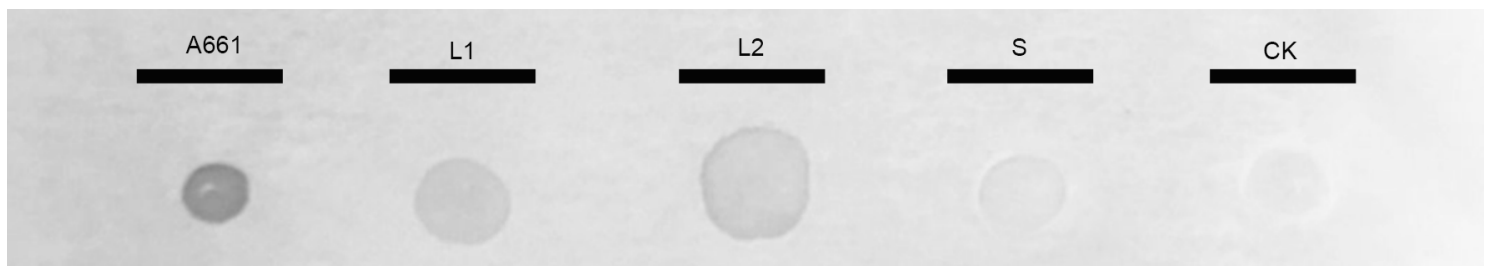

Fig. 2 Specificity tests using purified protein and tissue blot-ELISA. A661 indicates the purified TSHSV-HP4 protein. L1 and L2 indicate the lung proteins extracted from the wild TSHSV-positive turtles and laboratory TSHSV-infected turtles, respectively. $\mathrm{S}$ indicates the serum protein extracted from the laboratory TSHSV-infected turtles. CK indicates the lung protein extracted from healthy turtles.
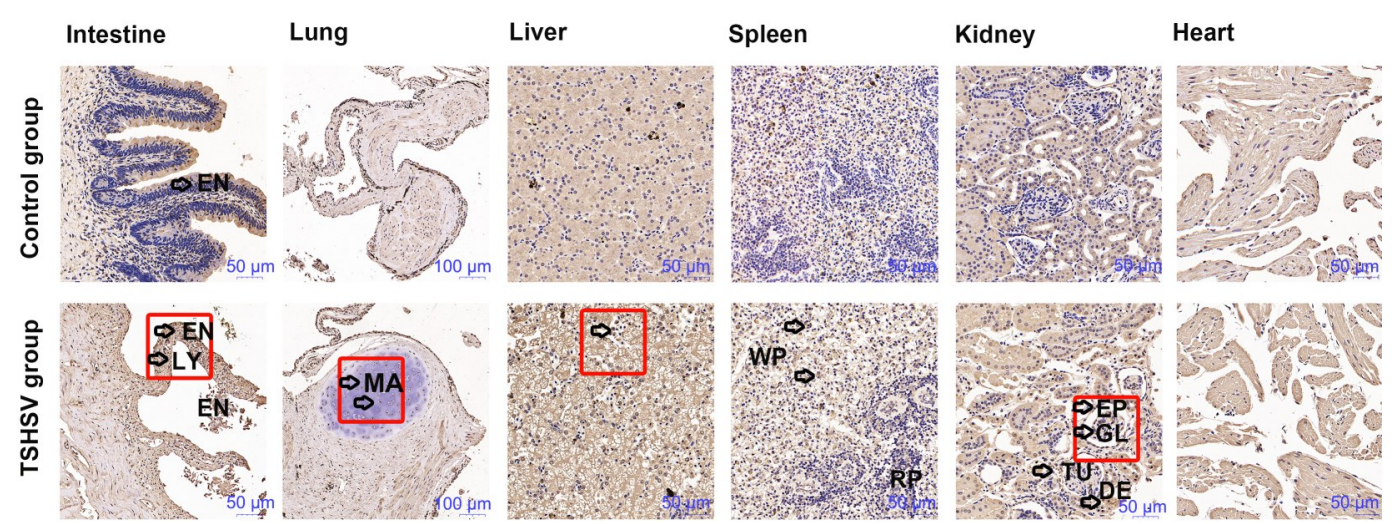

Fig. 3 Viral localization in different organs analyzed by IHC. Tissue areas framed in red show positive staining of viral protein. Positive signal was observed in lymphocytes (LY) and epibiotic enterocytes (EN) in the intestinal villi. Strong positive signal covered the nucleus of macrophages (MA) in the lung tissue. Prominent positive signal completely covered the hepatocyte nuclei, resembling nuclear immunostaining. Clear signal was observed in the white pulp (WP), but not the red pulp (RP), of spleen cells. Clear signal of TSHSV protein was evident in the glomerulus (GL) and epithelial cells (EP), while sporadic signals were observed within the lumen of tubules (TU) and necrotic debris (DE). Negative signal was observed in cardiac muscle fibers of the ventricular myocardium in both TSHSV-infected and negative control turtles. 
red pulp, of the spleen cells. The cells in the kidneys and spleen showed peripheral condensation of chromatin, partition of cytoplasm and nucleus into membrane. In the liver, a prominent positive signal was observed in the hepatocytes: strong staining completely covered the cell nucleus, resembling nuclear immunostaining. Vacuolar degeneration occurred in the hepatocytes simultaneously. In the heart, no signal was observed in the cardiac muscle fibers of the ventricular myocardium in either TSHSV-infected turtles or negative control turtles. Similar to the hepatocytes, a strong positive signal covered the nucleus of macrophages found in lung tissues.

\subsection{Clinicopathologic analysis of TSHSV-infected lung}

Histological examination revealed serious pathological changes in the lungs of TSHSV-infected turtles (Fig. 4). Additional lesions observed were severe interlobular oedema (Fig. 4a-III), acute necrotic bronchiolitis along with inflammatory cell infiltration, and large amounts of fibrin in the alveolar lumen (Fig. 4a). Acute hemorrhages were evident in the lung tissue, with substantial erythrocyte diapedesis (Fig. 4a-IV) in samples from the infected group compared with those from the control group (Fig. 4b-IV).
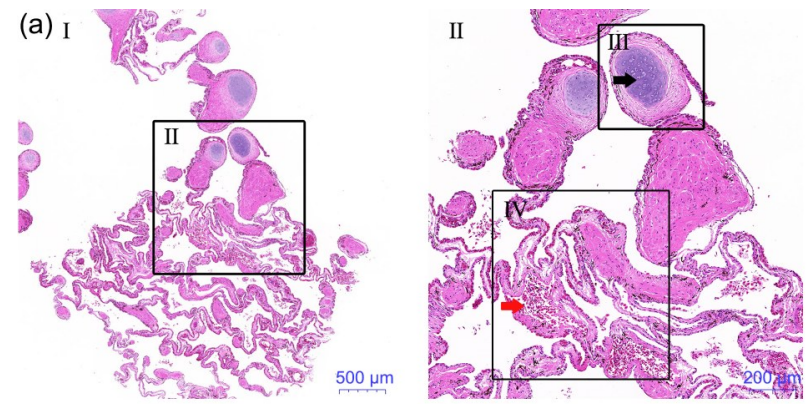

(b) I
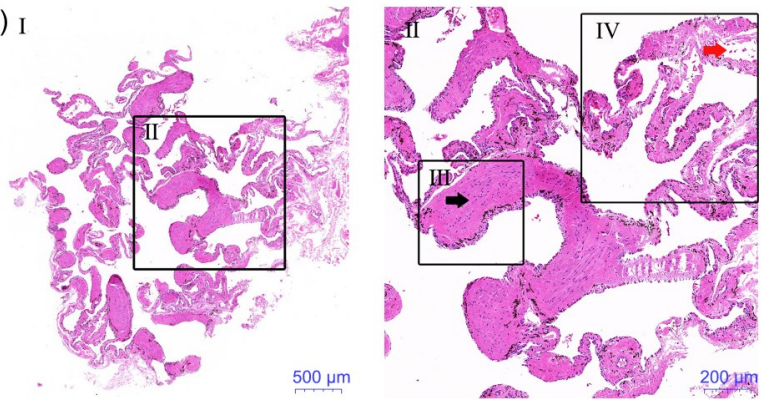

\subsection{Effects of anti-TSHSV-HP4 polyclonal antibody on immune genes of $T$. sinensis}

Antiviral genes including $M X 2$ and $R S A D 2$ were detected in various organs in turtles post-infection with crude virus and antibody-virus mixture. TSHSV messenger RNA (mRNA) copies were detected in all organs tested, including the lung, liver, kidney, spleen, and heart, both in the virus group and the virus-antibody group (Fig. 5a).

The viral mRNA copy number was higher in the virus-antibody group than in the virus group, especially in the lung, liver, and heart. The viral load difference between the two groups was significant in the lung and heart $(P<0.05)$. Fewer viral mRNA copies were detected in the kidney and spleen of the virus-antibody group than in those of the virus group $(P>0.05$; Fig. 5a).

The relative mRNA expression of $M X 2$ was higher in all organs tested in the virus and virus-antibody groups than in those of the antibody and control groups. Significantly higher mRNA expression was detected in the lung and heart tissues from the virus-antibody group than in those from the virus group, antibody group, and control group $(P<0.05$; Fig. 5 b). Much higher mRNA expression of $R S A D 2$ was detected in all tissues examined, including
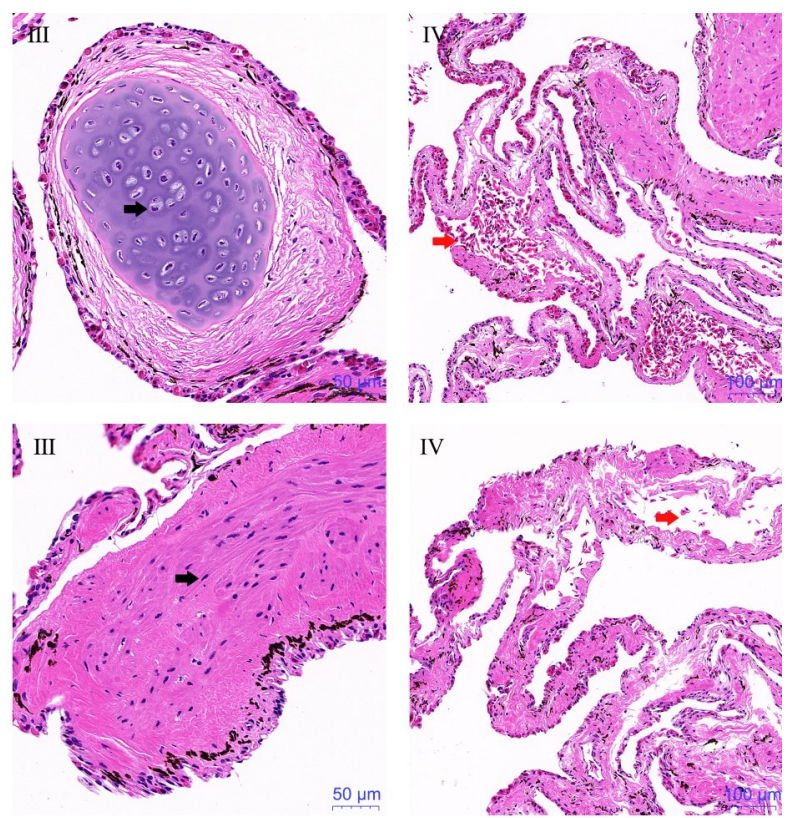

Fig. 4 Hematoxylin and eosin (H\&E) staining of TSHSV-infected lung tissue (a) and health lung tissue (b). Serious pathological changes occurred in the lungs. Significant macrophage infiltration observed in the lungs of a TSHSVinfected turtle (III). Acute hemorrhages were evident in the lungs, with erythrocyte diapedesis (IV). Red and black arrows indicate macrophages and erythrocytes, respectively. 
(a)

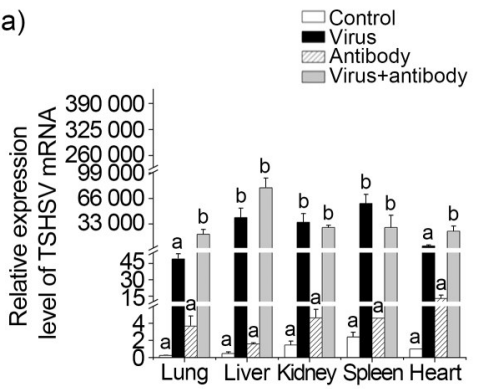

(b)

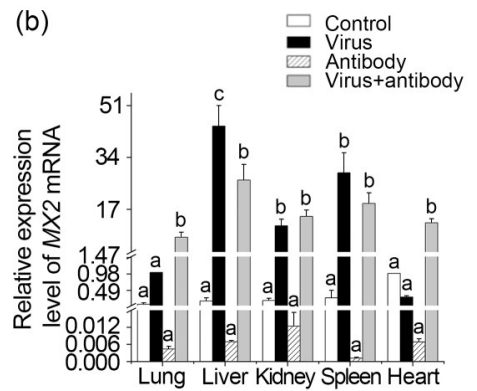

(c)

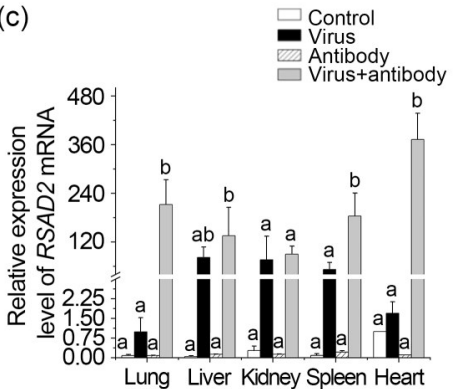

Fig. 5 qRT-PCR analysis of TSHSV and antiviral genes (MX2 and RSAD2). (a) TSHSV mRNA was detected in all organs tested, and the number of virus copies was higher in the lungs and heart of the antibody neutralizing group than in those of the virus group. The relative mRNA expression levels of $M X 2$ (b) and $R S A D 2$ (c) were higher in all organs tested in the virus and antibody neutralizing group than in those of the control and antibody groups. Each bar represents the mean value from five determinations including the standard error (SE). Different letters above the bars indicate significant differences $(P<0.05)$ among groups.

the lung, liver, spleen, kidney, and heart, in the virus group and virus-antibody group. Between these two groups, $R S A D 2$ levels were significantly upregulated in the lung, spleen, and heart in the virus-antibody group $(P<0.05$; Fig. 5c).

\section{Discussion}

Hemorrhagic syndrome is one of the most economically significant viral diseases affecting the domesticated Chinese softshell turtle (T. sinensis) in China (Liu et al., 2015). The etiological agent of this disease, TSHSV, has been confirmed to be a member of the genus Arterivirus within the unclassified family Arteriviridae (Lyu et al., 2019). In this study, we determined the effects of polyclonal antibody against TSHSV in activating the immune system of $T$. sinensis. Furthermore, we investigated the localization and tissue tropism of TSHSV in virus-infected organs.

In our previous work, TSHSV-HP4 was shown to have replicase activity since two domains, namely a Ploop containing a nucleoside triphosphate hydrolase domain and an EndoU-like endoribonuclease domain (Lyu et al., 2019). In this study, we successfully generated a partial protein of TSHSV-HP4 expressed as a recombinant $6 \times$ His-tagged protein of $44.3 \mathrm{kDa}$. In the western blot assay and dot-ELISA, the polyclonal protein serum reacted strongly to the purified recombinant TSHSVHP4 protein, suggesting its suitability as an antibody for the detection of TSHSV protein in a competitive ELISA format. In previous research, Liu et al. (2015) revealed that the highest levels of viral nucleic acids were found in the lung tissues of TSHSV-infected $T$. sinensis. Thus, the lungs of infected turtles were chosen as the target organ to further confirm the validity of TSHSV detection. Based on tissue blot-ELISA, the prepared antiserum reacted strongly with TSHSV using the crude proteins extracted from lung tissues of wild and laboratory TSHSV-infected $T$. sinensis, while a moderate signal was detected in their serum, which was consistent with the previous findings (Liu et al., 2015).

IHC was performed to investigate viral localization and tissue tropism in six different organs. The results showed that the virus was clearly seen localized in all these organs except the heart. A strong positive signal of virus protein was detected in the small intestine, liver, and kidney, and a weak signal in the lung, spleen, and heart (Fig. 3). These findings contrast with those of previous research, in which Liu et al. (2015) detected a higher level of virus titration in the lung, spleen, kidney, and heart by qRT-PCR. These conflicting results may be explained by the use of different methods in the two studies. The IHC assay used in this study complemented the viral localization and replication in conjunction with previous qRT-PCR results (Park et al., 2019; Qadiri et al., 2019). In the small intestine, a strong positive signal was observed specifically in intestinal villi, but not in the intestinal wall (Fig. 3), suggesting that the virus showed a tropism for lymphocytes and epibiotic enterocytes in the intestinal villi. This could explain the relatively low virus titration detected in the intestine in the study by Liu et al. (2015), in which the whole small intestine was used for RNA extraction and virus titration detection. In addition, lymphocytic infiltration, abscission, and necrocytosis of the 
mucous epithelial cells implied a strong inflammatory reaction in the intestine (Fig. 3). In the lungs, a strong positive signal was observed in the nucleus of aggregated pulmonary alveolar macrophages located in the pulmonary aloveoli (Fig. 3), indicating that the virus was located in the lung tissues, and that active viral replication occurred simultaneously in pulmonary alveolar macrophages. In the case of arteriviruses, such as the PRRSV and EAV, macrophages have been shown to be the primary target cells for viral replication both in vivo and in vitro (Tirado and Yoon, 2003). Based on the histological analysis, the occurrence of acute necrotic bronchiolitis and erythrocyte diapedesis indicated a serious inflammatory response and hemorrhage (Fig. 4), similar to that observed in animals infected with other arteriviruses. Kvisgaard et al. (2017) found that ecchymotic hemorrhages were present in the dorsal lung lobes in PRRSV-infected swine. Vairo et al. (2014) also found that isolate $08 P 178$ of EAV can cause congestion, oedema, multifocal atelectasis, and lymphocytic infiltration of the perivascular and alveolar septa in the cranial lobe of the right lung of infected animals. In the liver, viral localization was restricted to the hepatic blood vessels with a prominent positive signal observed in the hepatocytes. A strong staining that completely covered the cell nucleus, resembling nuclear immunostaining, and virus replication were observed (Fig. 4). Conversely, Liu et al. (2015) detected a lower level of viral nucleic acids in the liver using qRT-PCR. This suggests that assembled viral particles are less localized in the liver during active virus replication. In addition, serious vacuolar degeneration was seen in the hepatocytes, consistent with our previous histological research (Liu et al., 2017). In the kidneys, a clear signal of virus was evident in the glomerulus and epithelial cells, while sporadic signals were observed within the lumen of tubules and in the necrotic debris, indicating a viral tropism for endothelial cells. This indicated that active viral replication and viral localization were present in these organs. Although the spleen and heart were known as organs containing a higher virus titer, we found a weak signal of virus protein in the white pulp and red pulp of the spleen, and in cardiac muscle fibers (Fig. 3), suggesting relatively inactive virus replication in these organs.

Based on the qRT-PCR analysis, relatively high mRNA expression of the interferon-stimulated genes (ISGs) $M X 2$ and RSAD2 was detected in all organs tested in the virus group and the antibody neutralizing group. MX2 protein of the family of dynamin-like GTPases is tightly regulated by type I and type III interferons in animals infected by the virus (Meier et al., 2018). This protein has been verified to have a high level of anti-viral activity and targets multiple viruses including arterivirus (Wang et al., 2016). For PRRSV, Wang et al. (2016) found, that overexpression of monkey $M x 2(m M x 2)$ suppressed PRRSV replication in a dosedependent manner, indicating its antiviral activity. Potential antiviral mechanisms of $M X 2$ against other viruses were also found, including binding on the nucleocapsid, thereby preventing viral mRNA synthesis and sequestration of viral protein (Meier et al., 2018). Similarly, RSAD2 has been demonstrated to be highly upregulated upon viral infection and has broad antiviral activity against PRRSV, measles virus, influenza virus, respiratory syncytial virus, and other viruses through a common inhibition effect at the virion release step (Wang et al., 2007; McGillivary et al., 2013; Fang et al., 2016; Kurokawa et al., 2019). In this study, RSAD2 mRNA expression was highly upregulated in the virus group and antibody neutralizing group compared with the control group and the antibody group, consistent with our previous research (Lyu et al., 2020). Interestingly, in the antibody neutralizing group, significantly higher expression of $M X 2, R A S D 2$, and TSHSV mRNA was detected in the lung and heart than in those of the virus group. A faster progression of this disease occurred in the antibody neutralizing group (data not shown), indicating the possibility of antibodydependent enhancement (ADE) of TSHSV. In addition, a higher virus titer was detected in the lung and heart of the virus antibody group than in those of the virus group. These organs are the two target organs of TSHSV. We speculate that ADE might be responsible for the higher expression of interferon (INF)-related genes and the higher virus titer in these two organs compared with those in the virus group. For dengue virus, both Dejnirattisai et al. (2010) and Si et al. (2016) claimed that polyclonal proteins to the specific viral structural protein were highly cross-reactive among dengue virus serotypes. Even at high concentrations, they did not neutralize infection, but potently promoted ADE. In previous studies, the lungs, especially the lung macrophages, have been identified as the major target responsible for the increased virus replication and injury concerns associated with ADE (Yip et al., 
2014; Wen et al., 2020). Wan et al. (2020) proved that high titers of severe acute respiratory syndrome coronavirus 1 (SARS-CoV-1) antibodies were associated with lung injury by inducing hyperimmunity through interactions of the antibody and $\mathrm{Fc}$ receptor $(\mathrm{FcR})$, which also help the virus to enter macrophages and subsequently replicate. Liu et al. (2019) also found a positive correlation between the antibody and the degree of pathological injury in the lungs. For group B coxsackievirus (CVB), it was evident that pre-existing antibody increased virus infection, which would be responsible for increasing the extent of cardiac injury. Increasing the amount of virus reaching the heart leads to a worsening of the lesions (Girn et al., 2002).

In conclusion, we successfully cloned a partial TSHSV-HP4 protein and prepared the corresponding polyclonal antibody, which showed specific binding to the TSHSV proteins in the organs from viral-infected turtles. IHC technique determined that the virus was localized in various cells, including intestinal lymphocytes, enterocytes, kidney epithelial cells, spleen cells, and lung macrophages. In addition, two ISGs, namely $R S A D 2$ and $M X 2$, were highly upregulated after viral infection, and ADE seemed to occur post-stimulation by polyclonal antibody against this virus. Overall, these results are important in expanding our understanding of cell localization and the immune response of TSHSV in infected turtles.

\section{Acknowledgments}

This work was supported by the Zhejiang Provincial Science and Technology Program (Nos. 2020YSZX003 and 2020YSZX010) and the Zhejiang Provincial Science Exploratory Program (No. 2019TSX01), China. We appreciated the Huzhou Technical Advice Station (Huzhou, China) for the provision of infected Trionyx sinensis.

\section{Author contributions}

Haiqi ZHANG and Li LIU designed the project. Sunjian LYU and Xuemei YUAN performed the experiments, analyzed the data, and wrote the paper. Zhe YU, Weida SHI, and Yinglei WU cultured the turtles. Xiaoying HANG corrected the paper. All authors have read and approved the final manuscript. Therefore, all authors have full access to all the data in the study and take responsibility for its integrity and security.

\section{Compliance with ethics guidelines}

Sunjian LYU, Xuemei YUAN, Li LIU, Haiqi ZHANG, Zhe YU, Xiaoying HANG, Weida SHI, and Yinglei WU declare that they have no conflict of interest.
All institutional and national guidelines for the care and use of laboratory animals were followed.

\section{References}

Chen Z, Zhang MH, Zhou XP, et al., 2017. Development and detection application of monoclonal antibodies against Zucchini yellow mosaic virus. J Integr Agric, 16(1): 115-124. https://doi.org/10.1016/s2095-3119(16)61416-8

Dejnirattisai W, Jumnainsong A, Onsirisakul N, et al., 2010. Cross-reacting antibodies enhance dengue virus infection in humans. Science, 328(5979):745-748. https://doi.org/10.1126/science.1185181

Fang JY, Wang HY, Bai J, et al., 2016. Monkey viperin restricts porcine reproductive and respiratory syndrome virus replication. PLoS ONE, 11(5):e0156513. https://doi.org/10.1371/journal.pone.0156513

Fang Y, Snijder EJ, 2010. The PRRSV replicase: exploring the multifunctionality of an intriguing set of nonstructural proteins. Virus Res, 154(1-2):61-76. https://doi.org/10.1016/j.virusres.2010.07.030

Fishery Administration Bureau of Ministry of Agriculture and Rural Areas, National Aquatic Technology Extension Station, China Society of Fisheries, 2018. China Fishery Statistical Yearbook. China Agriculture Press, Beijing, China, p.24 (in Chinese).

Girn J, Kavoosi M, Chantler J, 2002. Enhancement of coxsackievirus B3 infection by antibody to a different coxsackievirus strain. J Gen Virol, 83(2):351-358. https://doi.org/10.1099/0022-1317-83-2-351

Knoops K, Kikkert M, van der Worm SHE, et al., 2008. SARScoronavirus replication is supported by a reticulovesicular network of modified endoplasmic reticulum. PLoS Biol, 6(9):e226. https://doi.org/10.1371/journal.pbio.0060226

Kurokawa C, Iankov ID, Galanis E, 2019. A key anti-viral protein, RSAD2/VIPERIN, restricts the release of measles virus from infected cells. Virus Res, 263:145-150. https://doi.org/10.1016/j.virusres.2019.01.014

Kvisgaard LK, Larsen LE, Hjulsager CK, et al., 2017. Genetic and biological characterization of a Porcine Reproductive and Respiratory Syndrome Virus 2 (PRRSV-2) causing significant clinical disease in the field. Vet Microbiol, 211:74-83. https://doi.org/10.1016/j.vetmic.2017.10.001

Liu L, Cao Z, Lin F, et al., 2015. Partial sequence of a novel virus isolated from Pelodiscus sinensis hemorrhagic disease. Intervirology, 58(4):197-204. https://doi.org/10.1159/000437354

Liu L, Cao Z, Lin F, et al., 2017. The histopathological characteristics caused by Trionyx sinensis hemorrhagic syndrome virus (TSHSV) and comparative proteomic analysis of liver tissue in TSHSV-infected Chinese softshelled turtles (Pelodiscus sinensis). Intervirology, 60(1-2): 19-27. https://doi.org/10.1159/000479795 
Liu L, Wei Q, Lin QQ, et al., 2019. Anti-spike IgG causes severe acute lung injury by skewing macrophage responses during acute SARS-CoV infection. JCI Insight, 4(4): e123158.

https://doi.org/10.1172/jci.insight.123158

Lyu SJ, Yuan XM, Zhang HQ, et al., 2019. Complete genome sequence and analysis of a new lethal arterivirus, Trionyx sinensis hemorrhagic syndrome virus (TSHSV), amplified from an infected Chinese softshell turtle. Arch Virol, 164(10):2593-2597.

https://doi.org/10.1007/s00705-019-04324-8

Lyu SJ, Yuan XM, Zhang HQ, et al., 2020. Transcriptome profiling analysis of lung tissue of Chinese soft-shell turtle infected by Trionyx sinensis Hemorrhagic Syndrome Virus. Fish Shellfish Immunol, 98:653-660. https://doi.org/10.1016/j.fsi.2019.10.061

McGillivary G, Jordan ZB, Peeples ME, et al., 2013. Replication of respiratory syncytial virus is inhibited by the host defense molecule viperin. $J$ Innate Immun, 5(1):60-71. https://doi.org/10.1159/000342473

Meier K, Jaguva Vasudevan AA, Zhang ZL, et al., 2018. Equine MX2 is a restriction factor of equine infectious anemia virus (EIAV). Virology, 523:52-63. https://doi.org/10.1016/j.virol.2018.07.024

Park H, Jung S, Shin H, et al., 2019. Localization and persistence of hepatitis A virus in artificially contaminated oysters. Int J Food Microbiol, 299:58-63. https://doi.org/10.1016/j.ijfoodmicro.2019.03.017

Qadiri SSN, Kim SJ, Krishnan R, et al., 2019. Localization and tissue tropism of viral haemorrhagic septicemia virus (VHSV) in experimentally infected juvenile olive flounder, Paralichthys olivaceus: an in situ hybridization and immunohistochemical study. Aquaculture, 505:242-252. https://doi.org/10.1016/j.aquaculture.2019.02.061
Si LL, Guo XL, Guo PJ, et al., 2016. Neutralizing effect and antibody dependent enhancement activity of anti-DENV prM polyclonal antibody. J Sun Yat-Sen Univ (Med Sci), 37(3):321-325 (in Chinese).

https://doi. org/10.13471/j. cnki. j. sun. yat-sen. univ(med. sci). 2016.0057

Tirado SMC, Yoon KJ, 2003. Antibody-dependent enhancement of virus infection and disease. Viral Immunol, 16(1):69-86. https://doi.org/10.1089/088282403763635465

Vairo S, Saey V, Bombardi C, et al., 2014. The recent European isolate $(08 \mathrm{P} 178)$ of equine arteritis virus causes inflammation but not arteritis in experimentally infected ponies. J Comp Pathol, 151(2-3):238-243. https://doi.org/10.1016/j.jcpa.2014.04.008

Wan YS, Shang J, Sun SH, et al., 2020. Molecular mechanism for antibody-dependent enhancement of coronavirus entry. $J$ Virol, 94(5):e02015-19. https://doi.org/10.1128/JVI.02015-19

Wang HY, Bai J, Fan BC, et al., 2016. The interferon-induced $\mathrm{Mx} 2$ inhibits porcine reproductive and respiratory syndrome virus replication. J Interferon Cytokine Res, 36(2):129-139. https://doi.org/10.1089/jir.2015.0077

Wang XY, Hinson ER, Cresswell P, 2007. The interferoninducible protein viperin inhibits influenza virus release by perturbing lipid rafts. Cell Host Microbe, 2(2):96-105. https://doi.org/10.1016/j.chom.2007.06.009

Wen JQ, Cheng YF, Ling RS, et al., 2020. Antibodydependent enhancement of coronavirus. Int J Infect Dis, 100:483-489. https://doi.org/10.1016/j.ijid.2020.09.015

Yip MS, Leung NHL, Cheung CY, et al., 2014. Antibodydependent infection of human macrophages by severe acute respiratory syndrome coronavirus. Virol J, 11:82. https://doi.org/10.1186/1743-422X-11-82 